\title{
THE INFRARED ARC SPECTRA OF MANGANESE AND RHENIUM
}

\author{
By William F. Meggers
}

ABSTRACT

New types of photographic plates sensitive to infrared radiation are employed to explore the long-wave portions of manganese and rhenium arc spectra from 7,200 to $11,000 \mathrm{~A}$. The sources were metallic manganese in a copper are and metallic rhenium in a silver arc. The spectrograms were made with large concave grating spectrographs, the first half of the range being investigated with a threefold increase in dispersion and resolution. Many new lines have been recorded in each spectrum and these in turn have disclosed new spectral terms or atomic energy levels. Lines in both spectra exhibit hyperfine structure.

\section{CONTENTS}

I. Introduction

II. Apparatus and methods

III. Results

1. Manganese

2. Rhenium

\section{INTRODUCTION}

Metallic manganese was first isolated in 1774 and for 151 years remained the only known metal with a maximum valence of 7 . Nearly a century after its discovery, Mendeléeff predicted, on the basis of a periodic arrangement of the elements, that 2 homologues of manganese should exist. These hypothetical elements were referred to as eka-manganese and dwi-manganese, or more recently as atomic numbers 43 and 75 , until they were finally captured in 1925 by Noddack and Tacke, ${ }^{1}$ and renamed masurium and rhenium, respectively. At the present time the amount of concentrated masurium is probably still too small for the study of its optical spectra, so that manganese and rhenium are the only metals of Group VII a vailable for spectroscopic study.

Although the spectra of manganese have been under investigation since 1864 , they are still far from being in a satisfactory state, especially at their extremes. The longer wave portion of the manganese arc spectrum was investigated by Kiess and Meggers ${ }^{2}$ in 1920 with dicyanin sensitized emulsions but even with 5-hour exposure only four lines beyond $9,000 \mathrm{~A}$ were detected.

The first extensive description and analysis of the rhenium are spectrum was published by Meggers ${ }^{3}$ in 1931 , but the observations

1 W. Noddack and I. Tacke, Naturwissenschaften, vol. 13, p. 567, 1925.

C. C. Kiess and W. F. Meggers, B.S. Sci. Pap., vol. 16 (S372), p. 51, 1920

3 W. F. Meggers, B.S. Jour. Research, vol. 6 (R P322), p. 1027, 1931. See also W. Mreidinger, Zeitschr. \&. Phys., vol. 68, p. 331, 1931; H. Schober, Wiener Ber.IIa, vol. 140, pp. 79 and 629, 1931; vol. 141, p. 601, 1932; จol. 142, p. $35,1933$. 
did not extend beyond 8,798 $\mathrm{A}$ in the infrared. The long-wave limit of these observations was imposed in part by the scarcity of rhenium, but mainly by the relatively low infrared sensitivity of the best photographic materials then at hand.

During the past year new types of photographic plates sensitive to infrared radiation have become available. These improved materials now permit the use of increased dispersion in the range formerly studied, and what is still more important they advance the longwave limit of spectrographic observations to $11,000 \mathrm{~A}$, or even beyond. Such superior photographic emulsions have recently been employed in a study of the arc spectra of manganese and rhenium, and the results form the contents of this paper. These observations constitute improved and extended descriptions of the characteristic emission spectra, and analyses of the new data give additional information as to the spectral and atomic structure of systems with 7 valence electrons.

\section{APPARATUS AND METHODS}

The spectra here described were obtained by dispersing and photographing the light from electric arcs at atmospheric pressure, the arcs being operated on a d.c. circuit with applied potential of 220 volts and current of 6 to 8 amperes.

Metallic manganese of high purity was fused on copper rods and thus used for the production of the manganese arc spectrum. The manganese, kindly supplied by Dr. F. M. Walters, Jr., was prepared in the Bureau of Metallurgical Research, Carnegie Institute of Technology. In the spectral range examined only one impurity line $(\mathrm{Mg} 8,806 \mathrm{~A})$ was detected.

My former study of rhenium spectra (RP322) was executed with part of a gram of pure potassium perrhenate which was still very scarce early in 1930. However, in this same year, relatively large quantities of rhenium salts and metal were uncovered as a by-product of certain chemical industries ${ }^{4}$ and pure metallic rhenium in larger quantity became available for the first time. A sample of metallic rhenium from Die Vereinigte Chemische Fabriken zu Leopoldshall, Germany, was used in the present investigation. This metal was in the form of fine powder or sponge, and when a small portion of it was fused on silver electrodes in the electric arc, it served very satisfactorily in producing an intense spectrum. The only impurities detected in the infrared range were two lines belonging to potassium (7,664 and 7,699 A), two characteristic of sodium (8,183 and 8,194 A); and three due to ionized calcium $(8,498,8,542$, and $8,662 \mathrm{~A})$.

The spectrograms were made with concave gratings of 21.7 feet radius of curvature, the gratings being mounted so as to give stigmatic slit images in the manner described elsewhere. ${ }^{5}$ Two different gratings were used, one having 20,000 lines per inch and the other 7,500 lines per inch. The former covered the interval 7,200 to 9,000 A with a scale of $3.5 \mathrm{~A} / \mathrm{mm}$, while observations with the latter extended from 8,600 to $11,000 \mathrm{~A}$ with an average scale of $10.2 \mathrm{~A} / \mathrm{mm}$.

Several new types of photographic plates for spectrography developed by the Research Laboratory of the Eastman Kodak Co. were

4. Feit, Zeitschr. Angew. Chem., vol. 43, p. 459, 1930.

6 W. F. Meggers and Keivin Burns, B.S.Sci. Papers, vol. 18 (S441), p. 185, 1922. 
described about a year ago. ${ }^{6}$ Since that time still further improvements have been made by combining special types of emulsions with new photosensitizing dyes, so that the entire range of visible spectrum and an equal range of adjacent infrared is now easily recorded on commercial plates. From among various new types of plates now available three were chosen for the work in hand; they are identified as types I-R, I-P, and I-Q. The maximum sensitivities of these 3 plates occur at about $8,100,8,600$, and $9,700 \mathrm{~A}$, respectively, but the 3 bands extend sufficiently to cover the entire range $1 \mathrm{~nm} 7,000$ A to $12,000 \mathrm{~A}$. Each plate just before use was hypersensitized in a dilute ammonia bath, and immediately after exposure was developed in Eastman X-ray developer at about $16^{\circ} \mathrm{C}$.

The extraordinary sensitiveness of the $R$ and $P$ sensitized plates has permitted the use of a larger dispersion to 9,000 $\mathrm{A}$ than had ever been applied to these spectra before and the increased scale and resolution have revealed some new facts in addition to yielding improved wave lengths. Exposures of 10 to 30 minutes duration were made in the first-order spectrum, but the longer time always resulted in a severe over exposure in the region of maximum sensitiveness. In several such cases it was necessary to diminish the dense blackness of the spectrograms by chemical reduction until they became transparent enough for measurement.

Wave lengths of the infrared manganese and rhenium lines were derived from measurements relative to international standards in the iron arc spectrum which was recorded in the second order of the grating on each spectrogram. The adopted standards were doubled in value to serve as effective standards in the infrared. Each line was measured 4 or more times on 2 or more spectrograms. The probable error in wave length of a sharp line in the first range is estimated at about $\pm 0.01 \mathrm{~A}$, but the uncertainty may be several times as large for the remainder.

\section{RESULTS}

\section{MANGANESE (Mn, Z-25)}

Kayser ${ }^{7}$ has summarized all the spectroscopic data on manganese up to the year 1910, at which time the wave-length measurements did not extend beyond 6,022 A in the orange. A few years later the arc spectrum of manganese was remeasured on the international scale by Fuchs ${ }^{8}$ who published a table of wave lengths extending from $2,289.93$ to $7,069.855 \mathrm{~A}$. In 1920 Kiess and Meggers ${ }^{9}$ published a list of lines beginning at about the middle of the visible spectrum and running out into the near infrared. Relatively small dispersion and a low scale of intensities were used and it now appears that the last real line in this list has a wave length of $9,243 \mathrm{~A}$. The long wave are spectrum of manganese was investigated radiometrically by Randall and Barker ${ }^{10}$ who found 20 lines in the interval 11,378 to $17,608 \mathrm{~A}$. Furnace spectra of manganese were studied by King ${ }^{11}$ in the range 2,795 to $8,200 \mathrm{~A}$, but no lines were found beyond $6,500 \mathrm{~A}$.

6 C. E. K. Mees, J. Opt. Soc. Am., vol. 22, p. 204, 1932.

7 H. Kayser, Handbuch der Spectroscopie, vol. 5, pp. 726-771, S. Hirzel, Leipzig, 1910.

8 H. Fuchs, Zeitschr.f.w.Phot., vol. 14, pp. 239-248, 263-280, 1914.

${ }^{8}$ H. Fuchs, Zeitschr.f.w.Phot., vol. 14, pp. 239-248, 263-280, 1914. . Kiess and W. F. Meggers, B.S.Sci.Papers, vol. 16 (S.372), p. 51, 1920.

10 H. M. Randall and E. F. Barker, Astrophys.J., vol. 49, p. 57, 1919.

11 A. S. King, Astrophys.J., vol. 53, p. 133, 1921. 
Study of the atomic spectrum of manganese in the infrared is seriously handicapped by a molecular spectrum when the arc is operated at ordinary atmospheric pressure. Bands presumably due to oxides of manganese pervade the whole range of infrared which can be photographed and tend to obscure atomic lines of low intensity. Unfortunately the band heads are inconspicuous but the rotation structure of overlapping bands is so prominent and entangled that it is impossible to distinguish faint atomic lines from the background of fine lines due to bands. Consequently in the list presented below, many of the weaker lines may belong to bands and some true atomic lines may have been omitted. It will be worth while to reinvestigate this range of the manganese spectrum with a source in which the molecular spectrum is suppressed.

In spite of the troublesome presence of bands on my spectrograms, this investigation of the atmospheric arc spectrum of manganese has revealed some interesting features of the atomic spectrum and has led to some corrections and extensions of the spectral structure analysis.

The arc spectrum of manganese has played an important role in the development of a theoretical interpretation of complex spectra. Two series of triplets were recognized in this spectrum by Kayser and Runge ${ }^{12}$ nearly 40 years ago, but no further progress was made until 1922 when Catalán ${ }^{13}$ extended the analysis and drew attention to the existence of the certain groups of lines which he called multiplets. Up to this time only singlets, doublets, and triplets had been recognized in spectra, but now it appeared that spectral terms of higher multiplicity must also occur. This was indeed found to be the case and the discovery of quartet, quintet, sextet, etc., terms in other complex spectra soon led to a general interpretation of all atomic spectra. ${ }^{14}$ The correlation of Zeeman effects with multiplet structure ${ }^{15}$ showed that the two sets of threefold terms found in manganese in reality belonged to sextet and octet systems and thus removed an apparent violation of the alternation law. Manganese multiplets resulting from quartet terms were first pointed out by Russell ${ }^{16}$ and some additions to these were published by McLennan and McLay, ${ }^{17}$ who also gave the first comprehensive list of term values for the Mn I spectrum. This term table was copied by Bacher and Goudsmit ${ }^{18}$ in a recent compilation of atomic energy states with modern notation added.

The main features of the infrared spectrum of manganese can be stated as follows: The most prominent group of lines in the investigated range is the $y^{6} \mathrm{P}-b^{6} \mathrm{D}$ multiplet lying between $8,670.92$ and $8,740.93 \mathrm{~A}$. Each of the nine lines in this multiplet is now shown to be complex with hyperfine structure. The occurrence of hyperfine structure among manganese lines was first mentioned by Janicki ${ }^{19}$ who also recognized the structures to be of the type since called "flag patterns" in which both intensities and intervals of components decrease in one direction. Hyperfine structure in neutral manganese

\footnotetext{
${ }^{12}$ H. Kayser and C. Runge, Abh.Berl.Akad.; 1894.

13 M. A. Catalán, Phil.'Trans., vol. 223, pp. 127-173, 1922.

$14 \mathrm{H}$. Hund, Linienspektren und Periodisches System der Elemente, Julius Springer, Berlin, 1927.

$15 \mathrm{E}$. Back and A. Landé, Zeemaneffekt und Multiplettstruktur der Spektrallinien, Julius Springer, Berlin, 1925.

${ }_{16}^{16}$ H. N. Russell, Astrophys.J., vol. 61, p. 223, 1925.

17 J. C. McLennan and A. B. McLay, Trans.Roy. Soc. Canada, III, vol. 20, p. 15, 1926.

18 R. F. Bacher and S. Goudsmit, A tomic Energy States, McGraw Hill Book Co., 1932.

10 L. Janicki, Ann.d.Phys. (4), vol. 29, p. 833; 1909.
} 
was further investigated by White and Ritschl, ${ }^{20}$ who showed that this structure was determined primarily by the coupling between the $4 s$ electron and the atomic nucleus. This $4 s$ electron is present in both terms producing the $y^{6} \mathrm{P}^{\circ}-b^{6} \mathrm{D}$ multiplet, so that the complexity of the lines could have been predicted. Although the structures are not resolved in my grating spectrograms they are clearly of the flagpattern type with a maximum spread of components of about $0.5 \mathrm{~A}$.

Another prominent group of long-wave manganese lines is the $z^{4} \mathrm{~F}^{\circ}-b^{4} \mathrm{D}$ multiplet ranging from $7,680.22$ to $7,821.25 \mathrm{~A}$. Superposed on this group is another one, $z^{6} \mathrm{~F}^{\circ}-c^{6} \mathrm{D}$, part of the latter term having been found by Russell ${ }^{21}$ in his search for series in this spectrum. On account of the hazy character of the lines and rapid decline of intensity with quantum numbers, the weakest level $c^{6} \mathrm{D}_{\frac{1}{3}}$ cannot be established with certainty. Combinations of the strongest level $c^{6} \mathrm{D}_{4}$ point to a correction which is necessary in the $z^{6} \mathrm{~F}^{\circ}$ term. The level $z^{6} \mathrm{~F}_{5 \frac{1}{2}}=16,624.24$ appears to have been derived on the assumption that a line at $3,806.866 \mathrm{~A}$ represents the combination $a^{6} \mathrm{D}_{41 / 2} z^{6} \mathrm{~F}^{\circ}{ }_{5 / 2}$, but the neighboring line at $3,806.724 \mathrm{~A}$ is the proper one so that $z^{6} \mathrm{~F}^{\circ}{ }_{51 / 2}=$ $16,623.30$.

McLennan and McLay ${ }^{22}$ proposed a new ${ }^{6} \mathrm{P}$ term with levels at $6,668.12,6,626.07$, and $6,604.68$, but were suspicious of it because the level separations violated the interval rule, and combinations with $a^{6} \mathrm{D}$ violated the intensity rule. The correct values for these levels are now found to be those given in table 1 for $v^{6} \mathrm{P}^{\circ}$.

The only remaining group of strong lines occurs between 9,084.27 and $9,243.29 \mathrm{~A}$. It has been interpreted as the combination $a^{4} \mathrm{~F}-z^{4} \mathrm{D}^{\circ}$, thus establishing a new metastable term of the Mn I spectrum, and most likely arising from the $3 d^{7}$ configuration.

My results for the Mn I spectrum are presented in tables 1 and 2. Table 1 lists the new terms which have been identified, the values being relative to the normal state $a^{6} \mathrm{~S}=59,937.47$.

TABLE 1.-Energy levels of the manganese atom

\begin{tabular}{|c|c|c|c|}
\hline Symbol & $\mathrm{J}$ & Term value & $\mid \begin{array}{c}\text { Level } \\
\text { separation }\end{array}$ \\
\hline$a^{4} \mathrm{~F}$ & $\begin{array}{l}41 / 2 \\
31 / 2 \\
211 / 2 \\
11 / 2\end{array}$ & $\begin{array}{l}24,998.91 \\
24,896.20 \\
24,822.66 \\
24,772.57\end{array}$ & $\begin{array}{r}102.71 \\
73.54 \\
50.09\end{array}$ \\
\hline$z^{6} \mathrm{~F}^{\circ}$ & $51 / 2$ & $16,623.30$ & \\
\hline$v^{6} \mathrm{P}^{\circ}$ & $\begin{array}{l}31 / 2 \\
21 / 2 \\
11 / 2\end{array}$ & $\begin{array}{l}6,676.05 \\
6,645.99 \\
6,626.15\end{array}$ & $\begin{array}{l}30.06 \\
19.84\end{array}$ \\
\hline$c^{6} \mathrm{D}$ & $\begin{array}{r}41 / 2 \\
31 / 2 \\
21 / 2 \\
11 / 2 \\
11 / 2\end{array}$ & $\begin{array}{l}3,748.01 \\
3,581.25 \\
3,446.7 \\
3,369.5 \\
3,271.5 ?\end{array}$ & $\begin{array}{c}166.76 \\
134.5 \\
77.2 \\
97.0 ?\end{array}$ \\
\hline 'P? & $21 / 2$ & $3,475.46$ & \\
\hline
\end{tabular}

In table 2, estimated relative intensities, measured wave lengths, corresponding wave numbers in vacuum, and term combinations for the lines are shown in successive columns. Symbols accompanjing

${ }^{20}$ H. E. White and R. Ritschl, Phys.Rev., vol. 35, p. 1116, 1930

21 H. N. Russell, Astrophys.J., vol. 66, p. 287, 1927.

22 J. C. McLennan and A. B. McLay, Proc.Roy.Soc. Canada, III, vol. 20, p. 22, 1926 
the intensities have significance as follows: $\mathrm{c}=$ complex with hyperfine structure, $\mathrm{h}=$ hazy, $\mathrm{H}=$ very hazy, $\mathrm{l}=$ shaded to longer waves, $\mathrm{v}=$ shaded to violet, $\mathrm{n}=$ band head, $\mathrm{p}=$ part of band structure.

TABLE 2.-Infrared arc spectrum of manganese

\begin{tabular}{|c|c|c|c|}
\hline Intensity & $\lambda_{\text {sir }}$ I.A. & $\nu_{\mathrm{VAO}} \mathrm{cm}^{-1}$ & Level combination \\
\hline $\begin{array}{l}2 \mathrm{H} \\
2 \mathrm{p} ? \\
2 \mathrm{p} ? \\
2 \mathrm{p} ? \\
3\end{array}$ & $\begin{array}{l}10,622.5 \\
10,561.0 \\
10,537.8 \\
10,456.6 \\
10,436.7\end{array}$ & $\begin{array}{l}9,411.4 \\
9,466.2 \\
9,487.05 \\
9,560.7 \\
9,578.95\end{array}$ & $y^{4} \mathrm{P}_{21 / 2}^{\circ}-{ }^{4} \mathrm{P}_{21 / 2} ?$ \\
\hline $\begin{array}{c}3 \mathrm{~h} \\
4 \mathrm{~h} \\
8 \mathrm{~h} \\
2 \\
20 \mathrm{~h}\end{array}$ & $\begin{array}{l}10,316.0 \\
10,300.5 \\
10,212.34 \\
10,102.44 \\
10,052.9\end{array}$ & $\begin{array}{l}9,691.0 \\
9,705.6 \\
9,789.39 \\
9,895.89 \\
9,944.66\end{array}$ & $\begin{array}{l}x^{6} \mathrm{P}_{11 / 2}^{\circ}-d^{6} \mathrm{D}_{21 / 2} \\
x^{6} \mathrm{P}_{21 / 2}^{\circ}-d^{6} \mathrm{D}_{31 / 2} \\
x^{6} \mathrm{P}_{31 / 2}^{\circ}-d^{6} \mathrm{D}_{41 / 2}\end{array}$ \\
\hline $\begin{array}{l}5 \mathrm{~h} \\
3 \mathrm{p} ? \\
5 \mathrm{p} ? \\
5 \mathrm{p} ? \\
2 \mathrm{p} ?\end{array}$ & $\begin{array}{r}10,045.04 \\
9,822.68 \\
9,761.57 \\
9,753.24 \\
9,727.38\end{array}$ & $\begin{array}{r}9,952.44 \\
10,177.74 \\
10,241.45 \\
10,250.20 \\
10,277.44\end{array}$ & $x^{6} \mathrm{P}_{31 / 2}^{\circ}-d^{6} \mathrm{D}_{31 / 2}$ \\
\hline $\begin{array}{l}2 \mathrm{p} ? \\
3 \mathrm{p} ? \\
2 \mathrm{p} ? \\
15 \\
15\end{array}$ & $\begin{array}{l}9,719.17 \\
9,706.24 \\
9,703.56 \\
9,686.3 \\
9,684.9\end{array}$ & $\begin{array}{l}10,286.13 \\
10,299.83 \\
10,302.67 \\
10,321.0 \\
10,322.5\end{array}$ & $y^{4} \mathrm{P}_{11 / 2}^{\circ}-b^{4} \mathrm{D}_{11 / 2}$ \\
\hline $\begin{array}{l}40 \\
4 \mathrm{p} ? \\
6 \mathrm{p} ? \\
3 \mathrm{p} ? \\
3 \mathrm{p} ?\end{array}$ & $\begin{array}{l}9,676.50 \\
9,633.02 \\
9,631.02 \\
9,628.96 \\
9,626.10\end{array}$ & $\begin{array}{l}10,331.49 \\
10,378.12 \\
10,380.27 \\
10,382.49 \\
10,385.58\end{array}$ & $\begin{array}{l}y^{4} \mathrm{P}_{11 / 2}-b^{4} \mathrm{D}_{21 / 2} \\
z^{4} \mathrm{D}_{11 / 2}^{0}-{ }^{4} \mathrm{P}_{21 / 2} ?\end{array}$ \\
\hline $\begin{array}{l}3 \mathrm{p} ? \\
100 \mathrm{~h}\end{array}$ & $\begin{array}{l}9,624.04 \\
9,608.56\end{array}$ & $\begin{array}{l}10,387.80 \\
10,404.54\end{array}$ & $y^{4} \mathrm{P}_{21 / 2}^{\circ}-b^{4} \mathrm{D}_{31 / 2}$ \\
\hline $5 \mathrm{~h}$ & $9,606.71$ & $10,406.54$ & $\left\{\begin{array}{l}z^{4} \mathrm{D}_{11 / 2}^{\circ}-c^{6} \mathrm{D}_{21 / 2} \\
y^{4} \mathrm{P}_{1 / 2}^{\circ}-b^{4} \mathrm{D}_{1 / 2}\end{array}\right.$ \\
\hline $\begin{array}{l}3 \mathrm{p} ? \\
10 \mathrm{~h}\end{array}$ & $\begin{array}{l}9,598.7 \\
9,584.0\end{array}$ & $\begin{array}{l}10,415.2 \\
10,431.2\end{array}$ & $z^{4} \mathrm{D}_{21 / 2}^{1 / 2}-c^{6} \mathrm{D}_{31 / 2}^{1 / 2}$ \\
\hline $\begin{array}{r}20 \mathrm{~h} \\
10 \mathrm{~h} \\
5 \mathrm{~h} \\
8 \mathrm{~h} \\
4 \mathrm{~h}\end{array}$ & $\begin{array}{l}9,550.80 \\
9,542.14 \\
9,535.72 \\
9,502.12 \\
9,476.57\end{array}$ & $\begin{array}{l}10,467.46 \\
10,476.96 \\
10,484.01 \\
10,521.08 \\
10,549.45\end{array}$ & $\begin{array}{l}y^{4} \mathrm{P}_{11 / 2}^{\circ}-b^{4} \mathrm{D}_{11 / 2} \\
z^{4} \mathrm{D}_{11 / 2}^{1}-c^{6} \mathrm{D}_{11 / 2} \\
z^{4} \mathrm{D}_{21 / 2}^{0} \mathrm{P}_{21 / 2} \\
z^{4} \mathrm{D}_{21 / 2}^{0}-c^{6} \mathrm{D}_{21 / 2}^{0}\end{array}$ \\
\hline $\begin{array}{l}2 \mathrm{p} ? \\
40 \\
30 \mathrm{~h} \\
10 \mathrm{~h} \\
4 \mathrm{~h}\end{array}$ & $\begin{array}{l}9,474.9 \\
9,444.90 \\
9,429.58 \\
9,412.78 \\
9,408.38\end{array}$ & $\begin{array}{l}10,551.3 \\
10,584.82 \\
10,602.02 \\
10,620.94 \\
10,625.91\end{array}$ & $\begin{array}{l}y^{4} \mathrm{P}_{11 / 2}^{\circ}-b^{4} \mathrm{D}_{1 / 2} \\
y^{4} \mathrm{P}_{21 / 2}^{\circ}-b^{4} \mathrm{D}_{21 / 2} \\
z^{4} \mathrm{D}_{31 / 2}^{\circ}-c^{6} \mathrm{D}_{31 / 2}\end{array}$ \\
\hline $\begin{array}{c}40 \mathrm{~h} \\
20 \mathrm{~h} \\
5 \\
4 \mathrm{~h} \\
150\end{array}$ & $\begin{array}{l}9,336.47 \\
9,331.90 \\
9,325.16 \\
9,323.76 \\
9,243.29\end{array}$ & $\begin{array}{l}10,707.75 \\
10,712.99 \\
10,720.74 \\
10,722.35 \\
10,815.69\end{array}$ & $\begin{array}{l}z^{4} \mathrm{D}_{31 / 2}^{\circ}-4 \mathrm{P}_{21 / 2} ? \\
a^{4} \mathrm{~F}_{31 / 2}-z^{4} \mathrm{D}_{31 / 2}^{\circ} \\
y^{4} \mathrm{P}_{21 / 2}^{\circ}-b^{4} \mathrm{D}_{11 / 2}^{\circ} \\
a^{4} \mathrm{~F}_{43 / 2}-z^{4} \mathrm{D}_{31 / 2}^{\circ}\end{array}$ \\
\hline
\end{tabular}


TABLE 2.-Infrared arc spectrum of manganese-Continued

\begin{tabular}{|c|c|c|c|}
\hline Intensity & $\lambda_{\text {air }}$ I.A. & $\nu_{\mathrm{VBC}} \mathrm{cm}^{-1}$ & Level combinatiou \\
\hline $\begin{array}{c}4 \\
10 \\
3 \mathrm{p} ? \\
100 \\
2 \mathrm{p} ?\end{array}$ & $\begin{array}{l}9,240.81 \\
9,234.40 \\
9,220.36 \\
9,172.09 \\
9,164.77\end{array}$ & $\begin{array}{l}10, \$ 18.60 \\
10,826.11 \\
10,842.59 \\
10,899.66 \\
10,908.36\end{array}$ & $\begin{array}{l}a^{4} \mathrm{~F}_{3 L_{2}}-z^{4} \mathrm{D}_{21 / 2}^{2} \\
a^{4} \mathrm{~F}_{3 ! / 2} z^{4} \mathrm{D}_{2 L_{2}}^{2}\end{array}$ \\
\hline $\begin{array}{l}5 \\
2 \mathrm{p} ? \\
2 \\
50 \\
3 \mathrm{hn} ?\end{array}$ & $\begin{array}{l}9,155.85 \\
9,128.97 \\
9,125.0 \\
9,114.02 \\
9,101.2\end{array}$ & $\begin{array}{l}10,918.98 \\
10,951.14 \\
10,955.9 \\
10,969.10 \\
10,984.6\end{array}$ & $\begin{array}{l}a^{4} \mathrm{~F}_{11 / 2}-z^{4} \mathrm{D}_{\mathrm{i} / / 2} \\
a^{4} \mathrm{~F}_{21 / 2}-z \mathrm{D}_{1 / 2}^{\circ}\end{array}$ \\
\hline $\begin{array}{l}30 \\
2 \\
4 \mathrm{~h} \\
3 \mathrm{dp} ? \\
2\end{array}$ & $\begin{array}{l}9,084.29 \\
9,066.50 \\
9,021.80 \\
8,953.2 \\
8,932.96\end{array}$ & $\begin{array}{l}11,005.00 \\
11,026.59 \\
11,081.22 \\
11,166.1 \\
11,191.43\end{array}$ & $a^{4} \mathrm{~F}_{1,2 / 2}-z^{4} \mathrm{D}_{1 / 2}^{n}$ \\
\hline $\begin{array}{c}60 \mathrm{~h} \\
15 \mathrm{~h} \\
2 \mathrm{p} ? \\
4 \\
2 \mathrm{~h}\end{array}$ & $\begin{array}{l}8,929.72 \\
8,926.06 \\
8,901.0 \\
8,895.36 \\
8,859.08\end{array}$ & $\begin{array}{l}11,195.49 \\
11,200.08 \\
11,231.6 \\
11,238.72 \\
11,284.76\end{array}$ & $\begin{array}{l}x^{6} \mathrm{P}_{31 / 2}^{\circ}-c^{6} \mathrm{D}_{41 / 3} \\
x^{6} \mathrm{P}_{21 / 2}^{\circ}-c^{6} \mathrm{D}_{31 / 2} \\
x^{6} \mathrm{P}_{11 / 2}^{\circ}-c^{6} \mathrm{D}_{21 / 2}\end{array}$ \\
\hline $\begin{array}{l}3 \mathrm{~h} \\
2 \mathrm{p} ? \\
3 \\
4 \mathrm{~h} \\
3 \mathrm{~h}\end{array}$ & $\begin{array}{l}8,842.48 \\
8,834.70 \\
8,827.83 \\
8,820.26 \\
8,798.66\end{array}$ & $\begin{array}{l}11,305.94 \\
11,315.90 \\
11,324.70 \\
11,334.43 \\
11,362.25\end{array}$ & $\begin{array}{l}x^{6} \mathrm{P}_{21 / 2}^{\circ}-{ }^{-4} \mathrm{P}_{21 / 2} ? \\
x^{6} \mathrm{P}_{21 / 2}^{\circ}-c^{6} \mathrm{D}_{21 / 2} \\
x^{6} \mathrm{P}_{31 / 2}^{\circ}-c^{6} \mathrm{D}_{31 / 2}\end{array}$ \\
\hline $\begin{array}{c}6 \mathrm{~h} \\
5 \mathrm{~h} \\
1,000 \mathrm{cw} \\
300 \mathrm{c} \\
30 \mathrm{c}\end{array}$ & $\begin{array}{l}8,796.83 \\
8,767.96 \\
8,740.93 \\
8,737.32 \\
8,734.60\end{array}$ & $\begin{array}{l}11,364.61 \\
11,402.03 \\
11,437.29 \\
11,442.02 \\
11,445.58\end{array}$ & $\begin{array}{l}z^{4} \mathrm{D}_{21 / 2}^{\circ}-b^{4} \mathrm{D}_{31 / 2} \\
z^{4} \mathrm{D}_{11 / 2}^{11}-b^{4} \mathrm{D}_{21 / 2} \\
y^{6} \mathrm{P}_{31 / 2}^{\circ}-b^{6} \mathrm{D}_{41 / 2} \\
y^{6} \mathrm{P}_{31 / 2}^{\circ}-b^{6} \mathrm{D}_{31 / 2}^{\circ} \\
y^{6} \mathrm{P}_{31 / 2}^{\circ}-b^{6} \mathrm{D}_{21 / 2}\end{array}$ \\
\hline $\begin{array}{c}2 \mathrm{~h} \\
2 \mathrm{~h} \\
10 \\
500 \mathrm{cw} \\
300 \mathrm{c}\end{array}$ & $\begin{array}{l}8,729.80 \\
8,717.29 \\
8,710.21 \\
8,703.76 \\
8,701.05\end{array}$ & $\begin{array}{l}11,451.87 \\
11,468.31 \\
11,477.63 \\
11,486.14 \\
11,489.71\end{array}$ & $\begin{array}{l}z^{4} \mathrm{D}^{\circ}{ }_{1 / 2}-b^{4} \mathrm{D}_{11 / 2} \\
x^{6} \mathrm{P}_{31 / 2}^{\circ}-{ }^{4} \mathrm{P}_{21 / 2}\end{array}$ \\
\hline $\begin{array}{r}100 \mathrm{c} \\
2 \mathrm{~h} \\
200 \mathrm{c} \\
300 \mathrm{c} \\
200 \mathrm{c}\end{array}$ & $\begin{array}{l}8,699.13 \\
8,680.24 \\
8,673.97 \\
8,672.06 \\
8,670.92\end{array}$ & $\begin{array}{l}11,492.25 \\
11,517.26 \\
11,525.58 \\
11,528.12 \\
11,529.64\end{array}$ & $\begin{array}{l}y^{6} \mathrm{P}_{21 / 2}^{\circ}-b^{6} \mathrm{D}_{11 / 2} \\
z^{6} \mathrm{~F}_{31 / 2}^{\circ}-d^{6} \mathrm{D}_{31 / 2} \\
y^{6} \mathrm{P}_{1 / 12}^{\circ}-b^{6} \mathrm{D}_{21 / 2} \\
y^{6} \mathrm{P}_{1 / 2}^{\circ}-b^{6} \mathrm{D}_{11 / 2} \\
y^{6} \mathrm{P}_{1 / 1 / 2}^{0}-b^{6} \mathrm{D}_{1 / 2}\end{array}$ \\
\hline $\begin{array}{c}2 \mathrm{~h} \\
2 \mathrm{~h} \\
10 \mathrm{~h} \\
40 \mathrm{~h} \\
5\end{array}$ & $\begin{array}{l}8,666.3 \\
8,664.6 \\
8,659.38 \\
8,654.63 \\
8,603.03\end{array}$ & $\begin{array}{l}11,535.8 \\
11,538.0 \\
11,545.00 \\
11,551.34 \\
11,620.62\end{array}$ & $\begin{array}{l}z^{4} \mathrm{D}_{1 / 2}-b^{4} \mathrm{D}_{1 / 2} \\
z^{4} \mathrm{D}_{11 / 2}^{0}-b^{4} \mathrm{D}_{11 / 2} \\
z^{4} \mathrm{D}_{21 / 2}^{\circ}-b^{4} \mathrm{D}_{21 / 2} \\
z^{4} \mathrm{D}_{31 / 2}^{\circ}-b^{4} \mathrm{D}_{31 / 2}\end{array}$ \\
\hline $\begin{array}{r}2 \mathrm{H} \\
5 \mathrm{H} \\
8 \mathrm{~h} \\
10 \mathrm{~h} \\
1 \mathrm{H}\end{array}$ & $\begin{array}{l}8,602.1 \\
8,600.34 \\
8,558.63 \\
8,521.57 \\
8,506.0\end{array}$ & $\begin{array}{l}11,621.9 \\
11,624.26 \\
11,680.91 \\
11,731.71 \\
11,753.2\end{array}$ & $\begin{array}{l}z^{4} \mathrm{D}_{1 / 12}^{\circ}-b^{4} \mathrm{D}_{1 / 2} \\
z^{6} \mathrm{~F}_{5 / 2}^{\circ}-d^{6} \mathrm{D}_{41 / 2} \\
z^{4} \mathrm{D}_{21 / 2}^{\circ}-b^{4} \mathrm{D}_{1 / 2} \\
z^{4} \mathrm{D}_{31 / 2}^{\circ}-b^{4} \mathrm{D}_{21 / 3} \\
z^{4} \mathrm{~F}_{1,1 / 2}-c^{6} \mathrm{D}_{11 / 2}\end{array}$ \\
\hline
\end{tabular}


TABLE 2.-Infrared arc spectrum of manganese-Continued

\begin{tabular}{|c|c|c|c|}
\hline Intensity & $\lambda_{\text {air }}$ I.A. & $\nu_{\mathrm{V} 30} \mathrm{~cm}^{-1}$ & Level combination \\
\hline $\begin{array}{l}3 \\
1 \mathrm{H} \\
20 \mathrm{Hv} \\
2 \mathrm{H} \\
15 \mathrm{Hv}\end{array}$ & $\begin{array}{l}8,481.70 \\
8,476.3 \\
8,431.20 \\
8,421.12 \\
8,409.88\end{array}$ & $\begin{array}{l}11,786.85 \\
11,794.4 \\
11,857.45 \\
11,871.65 \\
11,887.51\end{array}$ & $\begin{array}{l}z^{4} \mathrm{~F}_{21 / 2}^{\circ}-c^{6} \mathrm{D}_{21 / 2} \\
b^{6} \mathrm{~S}_{21 / 2}-v^{6} \mathrm{P}_{31 / 2}^{\circ} \\
z^{4} \mathbf{F}_{21 / 2}^{\circ}-c^{6} \mathrm{D}_{11 / 2} \\
b^{6} \mathrm{~S}_{21 / 2}-v^{6} \mathrm{P}_{21 / 2}^{\circ}\end{array}$ \\
\hline $\begin{array}{l}10 \mathrm{Hv} \\
40 \\
4 \mathrm{~h} \\
2 \mathrm{H} \\
2\end{array}$ & $\begin{array}{l}8,395.87 \\
8,380.77 \\
8,373.93 \\
8,353.79 \\
8,344.80\end{array}$ & $\begin{array}{l}11,907.35 \\
11,928.80 \\
11,938.54 \\
11,967.33 \\
11,980.22\end{array}$ & $\begin{array}{l}b^{6} \mathrm{~S}_{21 / 2}-v^{6} \mathrm{P}_{1,1 / 2}^{\circ} \\
z^{4} \mathrm{~F}_{31 / 2}^{\circ}-c^{6} \mathrm{D}_{21 / 2}\end{array}$ \\
\hline $\begin{array}{l}5 \mathrm{Hl} \\
4 \mathrm{~h} \\
5 \mathrm{~h} \\
1 \\
1\end{array}$ & $\begin{array}{l}8,304.42 \\
8,284.48 \\
8,251.64 \\
8,237.87 \\
8,234.43\end{array}$ & $\begin{array}{l}12,038.48 \\
12,067.45 \\
12,115.48 \\
12,135.73 \\
12,140.80\end{array}$ & $\begin{array}{l}z^{4} \mathrm{~F}_{41 / 2}^{\circ}-c^{6} \mathrm{D}_{31 / 2} \\
a^{4} \mathrm{D}_{21 / 2}-y^{6} \mathrm{P}_{1 / 2}^{\circ}\end{array}$ \\
\hline $\begin{array}{c}2 \\
40 \mathrm{hl} \\
2 \\
2 \\
1\end{array}$ & $\begin{array}{l}8,229.99 \\
8,212.43 \\
8,210.16 \\
8,204.50 \\
8,194.06\end{array}$ & $\begin{array}{l}12,147.35 \\
12,173.32 \\
12,176.69 \\
12,185.09 \\
12,200.61\end{array}$ & $a^{4} \mathrm{D}_{21 / 2}-y^{6} \mathrm{P}_{21 / 2}^{\circ}$ \\
\hline $\begin{array}{l}2 \\
2 \\
3 \\
2 \\
3\end{array}$ & $\begin{array}{l}8,148.78 \\
8,119.36 \\
8,110.51 \\
8,105.89 \\
8,079.46\end{array}$ & $\begin{array}{l}12,268.41 \\
12,312.86 \\
12,326.30 \\
12,333.32 \\
12,373.67\end{array}$ & \\
\hline $\begin{array}{l}8 \\
5 \\
2 \\
1 \mathrm{~h} \\
4\end{array}$ & $\begin{array}{l}8,043.37 \\
8,032.54 \\
8,026.23 \\
8,021.69 \\
7,948.10\end{array}$ & $\begin{array}{l}12,429.18 \\
12,445.94 \\
12,455.73 \\
12,462.78 \\
12,578.17\end{array}$ & $a^{4} \mathrm{D}_{31 / 2}-y^{6} \mathrm{P}_{21 / 2}^{\circ}$ \\
\hline $\begin{array}{r}25 \\
2 \\
4 \\
2 \\
1 \mathrm{~h}\end{array}$ & $\begin{array}{l}7,942.91 \\
7,938.52 \\
7,928.45 \\
7,920.44 \\
7,916.31\end{array}$ & $\begin{array}{l}12,586.39 \\
12,593.35 \\
12,609.34 \\
12,622.10 \\
12,628.68\end{array}$ & $z^{4} \mathrm{~F}_{21 / 2}^{\circ}-b^{4} \mathrm{D}_{31 / 2}$ \\
\hline $\begin{array}{l}2 \\
1 \mathrm{~h} \\
2 \\
2 \\
3\end{array}$ & $\begin{array}{l}7,911.68 \\
7,889.61 \\
7,871.58 \\
7,865.36 \\
7,854.24\end{array}$ & $\begin{array}{l}12,636.07 \\
12,671.42 \\
12,700.44 \\
12,710.48 \\
12,728.48\end{array}$ & $z^{4} \mathrm{~F}_{11 / 2}^{\circ}-b^{4} \mathrm{D}_{21 / 2}$ \\
\hline $10 \mathrm{~h}$ & $7,834.34$ & $12,760.81$ & $\left\{\begin{array}{l}z^{6} \mathrm{~F}_{41 / 2}^{\circ}-c^{6} \mathrm{D}_{41 / 2} \\
z^{6} \mathrm{~F}^{\circ}\end{array}\right.$ \\
\hline $\begin{array}{l}20 \\
30 \mathrm{~h} \\
8 \mathrm{hd} ? \\
15 \mathrm{~h}\end{array}$ & $\begin{array}{l}7,821.25 \\
7,816.61 \\
7,806.00 \\
7,790.82\end{array}$ & $\begin{array}{l}12,782.17 \\
12,789.76 \\
12,807.14 \\
12,832.10\end{array}$ & $\begin{array}{l}z^{4} \mathrm{~F}_{31 / 2}^{21 / 2}-b^{4} \mathrm{D}_{31 / 2} \\
z^{4} \mathrm{~F}_{21 / 2}^{\circ}-b^{4} \mathrm{D}_{21 / 2} \\
z^{4} \mathrm{~F}_{\mathrm{i12}-2}^{\circ}-b^{4} \mathrm{D}_{11 / 2} \\
z^{6} \mathrm{~F}_{31 / 2}^{\circ}-c^{6} \mathrm{D}_{31 / 2}\end{array}$ \\
\hline $\begin{array}{c}1 \mathrm{~h} \\
250 \mathrm{hl} \\
20 \mathrm{Hl} \\
9 \mathrm{Hl} \\
4\end{array}$ & $\begin{array}{l}7,782.2 \\
7,764.72 \\
7,755.15 \\
7,752.67 \\
7,737.16\end{array}$ & $\begin{array}{l}12,846.3 \\
12,875.23 \\
12,891.11 \\
12,895.24 \\
12,921.09\end{array}$ & $\begin{array}{l}z^{6} \mathrm{~F}_{11 / 2}^{\circ}-c^{6} \mathrm{D}_{21 / 2} \\
z^{6} \mathrm{~F}_{51 / 2}^{\circ}-c^{6} \mathrm{D}_{41 / 2} \\
z^{4} \mathrm{~F}_{1 / 2}^{\circ}-b^{4} \mathrm{D}_{1 / 2} \\
z^{6} \mathrm{~F}_{21 / 2}^{1}-c^{6} \mathrm{D}_{21 / 2}\end{array}$ \\
\hline
\end{tabular}


TABLE 2.-Infrared arc spectrum of manganese-Continued

\begin{tabular}{|c|c|c|c|}
\hline Intensity & $\lambda_{\mathrm{Bir}} \mathrm{I} . \mathrm{A}$. & $\nu_{s} \mathrm{com}^{-1}$ & Level combination \\
\hline $\begin{array}{c}2 \mathrm{~h} \\
50 \mathrm{~h} \\
150 \mathrm{hl} \\
3 \mathrm{~h} \\
3\end{array}$ & $\begin{array}{l}7,735.77 \\
7,734.43 \\
7,733.24 \\
7,727.07 \\
7,721.57\end{array}$ & $\begin{array}{l}12,923.41 \\
12,925.66 \\
12,927.64 \\
12,937.96 \\
12,947.18\end{array}$ & $\begin{array}{l}z^{6} \mathrm{~F}_{11 / 2}^{\circ}-c^{6} \mathrm{D}_{1 L_{5}} \\
z^{4} \mathrm{~F}_{21 / 2}^{\circ}-b^{4} \mathrm{D}_{11 / 5} \\
z^{6} \mathrm{~F}_{11,5}^{\circ}-c^{6} \mathrm{D}_{31 / 5}\end{array}$ \\
\hline $\begin{array}{c}100 \mathrm{~h} \\
40 \mathrm{Hl} \\
10 \mathrm{Hl} \\
200 \\
2 \mathrm{Hl}\end{array}$ & $\begin{array}{l}7,712.42 \\
7,709.98 \\
7,706.52 \\
7,680.22 \\
7,677.46\end{array}$ & $\begin{array}{l}12,962.54 \\
12,966.64 \\
12,972.46 \\
13,016.88 \\
13,021.56\end{array}$ & 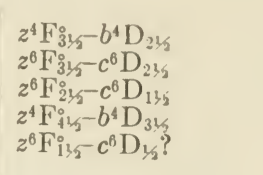 \\
\hline $\begin{array}{l}10 \mathrm{~h} \\
6 \mathrm{~h} \\
5 \mathrm{Hl} \\
7 \mathrm{Hl} \\
10 \mathrm{Hl}\end{array}$ & $\begin{array}{l}7,670.42 \\
7,667.89 \\
7,655.99 \\
7,651.62 \\
7,646.02\end{array}$ & $\begin{array}{l}13,033.51 \\
13,037.81 \\
13,058.08 \\
13,065.54 \\
13,075.11\end{array}$ & $\begin{array}{l}a^{8} \mathrm{~S}_{31 / 2}-a^{8} \mathbf{P}_{21 / 2}^{\circ} \\
a^{5} \mathrm{~S}_{31 / 2}-a^{8} \mathbf{P}_{31 / 5}^{3} \\
a^{8} \mathrm{~S}_{31 / 2}-a^{8} \mathbf{P}_{41 / 5}^{10}\end{array}$ \\
\hline $\begin{array}{l}3 \\
1 \\
2 \\
4 \\
3 \mathrm{~h}\end{array}$ & $\begin{array}{l}7,603.43 \\
7,552.10 \\
7,534.82 \\
7,515.74 \\
7,483.42\end{array}$ & $\begin{array}{l}13,148.34 \\
13,237.71 \\
13,268.07 \\
13,301.65 \\
13,359.20\end{array}$ & \\
\hline $\begin{array}{l}1 \mathrm{~h} \\
1 ? \\
1 ? \\
1 \\
3\end{array}$ & $\begin{array}{l}7,477.38 \\
7,453.88 \\
7,453.56 \\
7,446.16 \\
7,443.50\end{array}$ & $\begin{array}{l}13,370.00 \\
13,412.14 \\
13,412.72 \\
13,426.05 \\
13,430.85\end{array}$ & \\
\hline $\begin{array}{l}1 \\
2 \\
3 \\
2 \\
1\end{array}$ & $\begin{array}{l}7,432.27 \\
7,425.52 \\
7,415.81 \\
7,404.24 \\
7,391.73\end{array}$ & $\begin{array}{l}13,451.14 \\
13,463.37 \\
13,481.00 \\
13,502.06 \\
13,524.91\end{array}$ & \\
\hline $\begin{array}{l}1 \\
1 \\
2 \\
1 \\
1\end{array}$ & $\begin{array}{l}7,390.04 \\
7,387.15 \\
7,383.52 \\
7,380.88 \\
7,378.98\end{array}$ & $\begin{array}{l}13,528.01 \\
13,533.30 \\
13,539.95 \\
13,544.79 \\
13,548.28\end{array}$ & \\
\hline $\begin{array}{c}3 \\
3 \\
4001 \\
1 \\
2\end{array}$ & $\begin{array}{l}7,376.85 \\
7,342.80 \\
7,326.51 \\
7,322.22 \\
7,304.98\end{array}$ & $\begin{array}{l}13,552.19 \\
13,615.04 \\
13,645.31 \\
13,653.30 \\
13,685.53\end{array}$ & $y^{6} \mathrm{P}_{31 / 2}^{\circ}-a^{6} \mathrm{D} ?$ \\
\hline $\begin{array}{c}3001 \\
3 \mathrm{~h} \\
1 \\
2 \mathrm{~h}\end{array}$ & $\begin{array}{l}7,302.89 \\
7,301.38 \\
7,293.85 \\
7,287.10\end{array}$ & $\begin{array}{l}13,689.44 \\
13,692.27 \\
13,706.41 \\
13,719.10\end{array}$ & $y^{6} \mathrm{P}_{21 / 5}^{\circ}-a^{6} \mathrm{D} ?$ \\
\hline $\begin{array}{c}2501 \\
2 \\
15 \\
3\end{array}$ & $\begin{array}{l}7,283.80 \\
7,275.66 \\
7,247.82 \\
7,239.61\end{array}$ & $\begin{array}{l}13,725.32 \\
13,740.68 \\
13,793.46 \\
13,809.10\end{array}$ & $y^{6} \mathrm{P}_{1 / 2}^{\circ}-a^{6} \mathrm{D} ?$ \\
\hline
\end{tabular}




\section{RHENIUM $(\operatorname{Re}, Z=75)$}

My former description of the rhenium arc spectrum ${ }^{23}$ gave the wave lengths and estimated intensities of about 2,000 lines from $2,500.59$ in the ultraviolet to 8,797.6 in the infrared. About 500 of these lines, including nearly all of the stronger ones, were classified as combinations of established energy levels but relatively few of these levels could be grouped into identifiable terms. Now in this supplementary investigation of the the infrared, the data between 7,200 $\mathrm{A}$ and the former limit have been greatly improved and lines have been recorded out 10,639.44 A. Several hundred new lines have thus been added to the number characteristic of neutral rhenium atoms and additional spectral features of interest have been disclosed.

The use of higher dispersion and resolving power in the interval 7,200 to $9,000 \mathrm{~A}$ has given new data on hyperfine structure which is such a prominent feature of rhenium lines. Whereas the widest manganese lines cover an interval of only $0.5 \mathrm{~A}$, some of the infrared rhenium lines are nearly $2.0 \mathrm{~A}$ broad and are resolved into 5 or 6 components in the first order of our Rowland grating. Details of this hyperfine structure are reserved for another publication, but some qualitative information is shown here with the same symbols that were employed in Research Paper No. 322.

A brief analysis of the new infrared data disclosed five additional metastable levels in the $R e$ I spectrum. These are presented in table 3 , the values being relative to the normal state $a^{6} \mathrm{~S}=0.00$.

TABLE 3.-Energy levels of the rhenium atom

\begin{tabular}{|c|c|c|}
\hline $\begin{array}{c}\text { Level } \\
\text { no. }\end{array}$ & $\mathrm{J}$ & Value \\
\hline 17.1 & $21 / 2$ & $19,457.76$ \\
19.1 & $21 / 2$ & $23,154.75$ \\
20.1 & 312 & $24,724.12$ \\
20.2 & $21 / 2$ & $26,131.46$ \\
20.3 & $31 / 2$ & $27,141.00$ \\
\hline
\end{tabular}

Combinations of these levels with those previously described (RP 322) account for nearly all of the stronger lines in the infrared as well as many others in the remainder of the spectrum. No further attempts to establish middle or high levels have been made, this being postponed until the spectrum has been accurately described in the ultraviolet between 2,000 and 2,500 A. Furthermore, additional efforts to group levels into terms is regarded as premature until the Zeeman effect of rhenium lines has been extensively studied. It is obvious that the Re I spectrum exhibits a considerable number of wide-spreading, overlapping terms, and lacking confidence in the interval or intensity rules as criteria for grouping levels in such a case it may be hoped that the Zeeman effect and $g$-sum rule will really serve as a guide out of this wilderness. Comparison of the two spectra, Mn I and Re I, shows that the normal state (zero energy) is represented by a sextet $\mathrm{S}$ term in each and that both exhibit excited states belonging to quartet, sextet, and octet systems, but it would be presumptious to go beyond this at the present time. 
The new data for the infrared spectrum of rhenium are displayed in table 4 in which estimated relative intensities, measured wave lengths, vacuum wave numbers, and level combinations appear in successive columns. Fortunately molecular bands which are so troublesome in the Mn I spectrum are almost entirely absent from the Re I spectrum and it has, therefore, been possible to give a more satisfactory description of the latter.

TABLE 4.-Infrared arc spectrum of rhenium

\begin{tabular}{|c|c|c|c|c|c|c|c|}
\hline Intensity & $\lambda_{\text {sir I. A. }}$ & $\nu_{\mathrm{rac}} \mathrm{cml}^{-1}$ & $\begin{array}{l}\text { Level com- } \\
\text { bination }\end{array}$ & Intensity & $\lambda_{\text {air }}$ I. $A$. & $\nu_{\text {VAO }} \mathrm{cm}^{-1}$ & $\begin{array}{l}\text { Level com- } \\
\text { bination }\end{array}$ \\
\hline $\begin{array}{l}10 \mathrm{cW} \\
3 \\
5 \\
1 \\
1 \mathrm{cW}\end{array}$ & $\begin{array}{l}10,639.44 \\
10,625.57 \\
10,618.40 \\
10,609.28 \\
10,599.4\end{array}$ & $\begin{array}{l}9,396.42 \\
9,408.68 \\
9,415.03 \\
9,423.13 \\
9,431.92\end{array}$ & $\begin{array}{r}17.1-21 \\
6-20 \\
17.1-22\end{array}$ & $\begin{array}{l}20 \\
4 \\
6 \\
3 \\
1 \mathrm{cw} ?\end{array}$ & $\begin{array}{l}9,762.65 \\
9,752.07 \\
9,749.67 \\
9,748.60 \\
9,725.6\end{array}$ & $\begin{array}{l}10,240.31 \\
10,251.43 \\
10,253.95 \\
10,255.07 \\
10,279.3\end{array}$ & $\begin{array}{l}20.3-38 \\
19.1-26\end{array}$ \\
\hline $\begin{array}{l}4 \mathrm{cw} \\
4 \\
1 \\
3 \mathrm{c} \\
10 \mathrm{cW}\end{array}$ & $\begin{array}{l}10,556.52 \\
10,464.23 \\
10,440.92 \\
10,348.94 \\
10,332.57\end{array}$ & $\begin{array}{l}9,470.23 \\
9,553.74 \\
9,575.08 \\
9,660.18 \\
9,675.48\end{array}$ & $61-111$ & $\begin{array}{c}4 \\
50 \\
3 \\
1 \\
5 \mathrm{c}\end{array}$ & $\begin{array}{l}9,722.76 \\
9,710.52 \\
9,704.94 \\
9,685.69 \\
9,635.30\end{array}$ & $\begin{array}{l}10,282.33 \\
10,295.29 \\
10,301.21 \\
10,321.68 \\
10,375.66\end{array}$ & \\
\hline $\begin{array}{l}4 \mathrm{cw} \\
5 \mathrm{cw} \\
2 \\
1 ? \\
1\end{array}$ & $\begin{array}{l}10,287.58 \\
10,282.46 \\
10,262.77 \\
10,258.42 \\
10,242.68\end{array}$ & $\begin{array}{l}9,717.80 \\
9,722.63 \\
9,741.29 \\
9,745.42 \\
9,760.40\end{array}$ & $61-113$ & $\begin{array}{l}2 \ln ? \\
1 \\
3 \mathrm{cw} \\
2 \ln ? \\
8 \mathrm{c}\end{array}$ & $\begin{array}{l}9,617.78 \\
9,600.98 \\
9,591.32 \\
9,587.0 \\
9,581.12\end{array}$ & $\begin{array}{l}10,394.56 \\
10,412.75 \\
10,423.24 \\
10,427.9 \\
10,434.33\end{array}$ & 19. 1-28 \\
\hline $\begin{array}{l}4 \\
20 \\
2 \mathrm{cW} \\
1 \mathrm{c} ? \\
1 \mathrm{c} ?\end{array}$ & $\begin{array}{l}10,238.26 \\
10,206.32 \\
10,201.86 \\
10,197.58 \\
10,191.57\end{array}$ & $\begin{array}{l}9,764.61 \\
9,795.17 \\
9,799.45 \\
9,803.57 \\
9,809.35\end{array}$ & & $\begin{array}{l}6 \mathrm{cw} \\
1 \\
6 \mathrm{cWV} \\
3 \\
8\end{array}$ & $\begin{array}{l}9,571.75 \\
9,564.06 \\
9,523.40 \\
9,504.34 \\
9,500.49\end{array}$ & $\begin{array}{l}10,444.55 \\
10,452.94 \\
10,497.58 \\
10,518.62 \\
10,522.89\end{array}$ & \\
\hline $\begin{array}{c}4 \mathrm{c} \\
20 \mathrm{w} \\
100 \mathrm{cW} \\
1 \\
1 ?\end{array}$ & $\begin{array}{l}10,183.32 \\
10,175.68 \\
10,169.85 \\
10,162.75 \\
10,146.84\end{array}$ & $\begin{array}{l}9,817.29 \\
9,824.66 \\
9,830.30 \\
9,837.16 \\
9,852.59\end{array}$ & $\begin{array}{l}60-111 \\
60-112\end{array}$ & $\begin{array}{l}1 \mathrm{cW} \\
30 \\
1 \\
3 \\
15\end{array}$ & $\begin{array}{l}9,481.6 \\
9,470.14 \\
9,468.38 \\
9,427.53 \\
9,423.44\end{array}$ & $\begin{array}{l}10,543.9 \\
10,556.61 \\
10,558.57 \\
10,604.33 \\
10,608.93\end{array}$ & $\begin{array}{l}20.1-34 \\
20.3-39\end{array}$ \\
\hline $\begin{array}{c}2 \\
15 \\
1 \\
10 \mathrm{cW} \\
2 \mathrm{cw}\end{array}$ & $\begin{array}{l}10,138.24 \\
10,128.78 \\
10,127.21 \\
10,064.02 \\
10,059.56\end{array}$ & $\begin{array}{l}9,860.94 \\
9,870.16 \\
9,871.68 \\
9,933.66 \\
9,938.08\end{array}$ & & $\begin{array}{l}2 \ln ? \\
2 \\
40 \\
10 \mathrm{c} \\
1\end{array}$ & $\begin{array}{l}9,400.27 \\
9,391.12 \\
9,383.74 \\
9,380.24 \\
9,377.02\end{array}$ & $\begin{array}{l}10,635.08 \\
10,645.44 \\
10,653.81 \\
10,657.79 \\
10,661.45\end{array}$ & $26-75$ \\
\hline $\begin{array}{l}1 \\
5 \mathrm{c} \\
7 \mathrm{cW} \\
1 \mathrm{cWV} \\
1 \mathrm{c} ?\end{array}$ & $\begin{array}{l}10,056.81 \\
10,026.96 \\
10,019.21 \\
10,010.29 \\
10,006.4\end{array}$ & $\begin{array}{l}9,940.79 \\
9,970.38 \\
9,978.10 \\
9,986.99 \\
9,990.9\end{array}$ & $59-111$ & $\begin{array}{c}2 \\
1 \\
20 \mathrm{c} \\
2 \mathrm{H} ? \\
2 \mathrm{cW}\end{array}$ & $\begin{array}{l}9,370.87 \\
9,367.59 \\
9,363.13 \\
9,340.8 \\
9,337.73\end{array}$ & $\begin{array}{l}10,668.43 \\
10,672.18 \\
10,677.26 \\
10,702.8 \\
10,706.31\end{array}$ & \\
\hline $\begin{array}{l}1 \mathrm{c} ? \\
4 \\
1 \mathrm{c} \\
2 \\
60 \mathrm{cW}\end{array}$ & $\begin{array}{l}9,980.9 \\
9,976.45 \\
9,970.87 \\
9,964.90 \\
9,955.45\end{array}$ & $\begin{array}{l}10,016.4 \\
10,020.86 \\
10,026.47 \\
10,032.47 \\
10,042.00\end{array}$ & $58-111$ & $\begin{array}{l}2 \mathrm{cw} \\
1 \\
3 \\
5 \\
1\end{array}$ & $\begin{array}{l}9,332.47 \\
9,328.36 \\
9,325.90 \\
9,311.59 \\
9,307.72\end{array}$ & $\begin{array}{l}10,712.34 \\
10,717.06 \\
10,719.89 \\
10,736.36 \\
10,740.82\end{array}$ & \\
\hline $\begin{array}{c}3 \mathrm{cW} \\
200 \mathrm{cW} \\
20 \\
1 \\
1 \mathrm{cW} ?\end{array}$ & $\begin{array}{l}9,953.02 \\
9,949.90 \\
9,943.70 \\
9,937.8 \\
9,921.7\end{array}$ & $\begin{array}{l}10,044.45 \\
10,047.60 \\
10,053.86 \\
10,059.8 \\
10,076.2\end{array}$ & $58-112$ & $\begin{array}{l}1 \\
1 \mathrm{cW} \\
15 \mathrm{cW} \\
4 \\
2 \mathrm{cW}\end{array}$ & $\begin{array}{l}9,298.07 \\
9,278.3 \\
9,268.46 \\
9,262.28 \\
9,255.71\end{array}$ & $\begin{array}{l}10,751.97 \\
10,774.9 \\
10,786.32 \\
10,793.52 \\
10,801.18\end{array}$ & \\
\hline $\begin{array}{l}5 \mathrm{c} \\
6 \\
1 \\
2 \mathrm{cw} \\
15 \mathrm{vd} ?\end{array}$ & $\begin{array}{l}9,908.97 \\
9,903.30 \\
9,901.72 \\
9,884.10 \\
9,872.38\end{array}$ & $\begin{array}{l}10,089.10 \\
10,094.88 \\
10,096.49 \\
10,114.49 \\
10,126.50\end{array}$ & & $\begin{array}{r}1 \\
10 \\
4 \\
1 \\
2\end{array}$ & $\begin{array}{l}9,253.71 \\
9,250.02 \\
9,236.50 \\
9,220.40 \\
9,213.58\end{array}$ & $\begin{array}{l}10,803.52 \\
10,807.82 \\
10,823.65 \\
10,812.54 \\
10,850.57\end{array}$ & \\
\hline $\begin{array}{c}1 \\
20 \mathrm{cW} \\
9 \\
2 \\
1\end{array}$ & $\begin{array}{l}9,851.88 \\
9,842.63 \\
9,831.35 \\
9,813.75 \\
9,790.20\end{array}$ & $\begin{array}{l}10,147.57 \\
10,157.10 \\
10,168.76 \\
10,186.99 \\
10,211.50\end{array}$ & & $\begin{array}{l}2 \mathrm{cw} \\
2 \mathrm{cw} \\
1 \\
1 \\
1 \mathrm{cW}\end{array}$ & $\begin{array}{l}9,209.66 \\
9,205.12 \\
9,198.04 \\
9,192.18 \\
9,171.35\end{array}$ & $\begin{array}{l}10,855.19 \\
10,860.54 \\
10,868.90 \\
10,875.83 \\
10,900.53\end{array}$ & \\
\hline
\end{tabular}


TABLE 4.-Infrared arc spectrum of rhenium-Continued

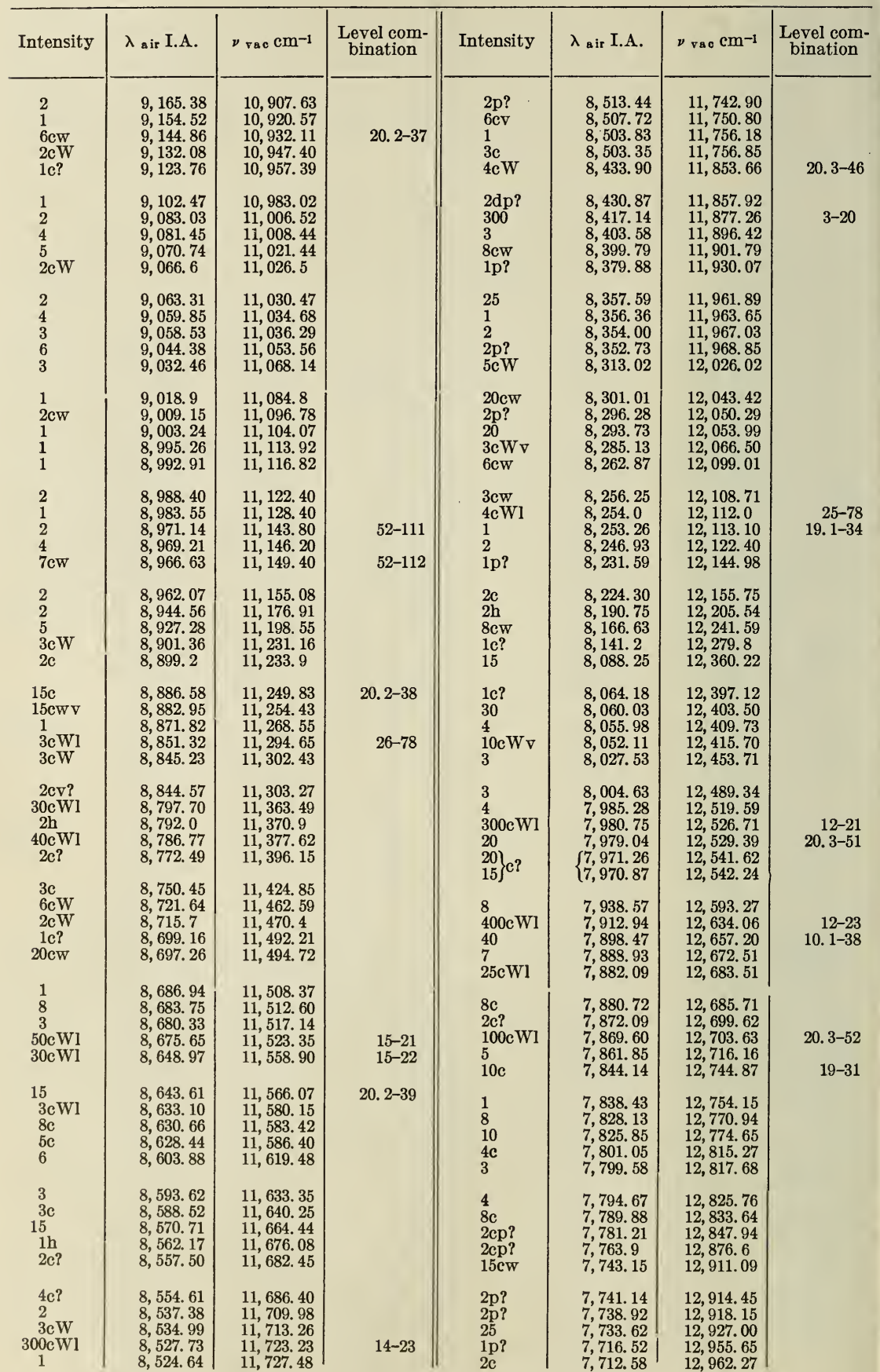


TABLE 4.-Infrared arc spectrum of rhenium-Continued

\begin{tabular}{|c|c|c|c|c|c|c|c|}
\hline Intensity & $\lambda_{\text {a ir }}$ I.A. & $\nu_{\mathrm{VAc}} \mathrm{cm}^{-1}$ & $\begin{array}{l}\text { Level com- } \\
\text { bination }\end{array}$ & Intensity & $\lambda_{\text {air }}$ I.A. & $\nu_{\mathrm{VAO}} \mathrm{cm}^{-1}$ & $\begin{array}{l}\text { Level com- } \\
\text { bination }\end{array}$ \\
\hline $\begin{array}{l}25 \mathrm{c} \\
20 \mathrm{c} \\
3 \\
2 \\
5 ?\end{array}$ & $\begin{array}{l}7,705.92 \\
7,693.63 \\
7,684.83 \\
7,683.45 \\
7,656.81\end{array}$ & $\begin{array}{l}12,973.47 \\
12,994.19 \\
13,009.07 \\
13,011.41 \\
13,056.68\end{array}$ & 20. 1-39 & $\begin{array}{l}4 \\
1 \mathrm{p} ? \\
1 \\
3 \\
2\end{array}$ & $\begin{array}{l}7,447.58 \\
7,446.97 \\
7,442.75 \\
7,440.71 \\
7,437.78\end{array}$ & $\begin{array}{l}13,423.49 \\
13,424.59 \\
13,432.20 \\
13,435.88 \\
13,441.20\end{array}$ & \\
\hline $\begin{array}{l}2 \mathrm{c} \\
400 \mathrm{cWl} \\
200 \mathrm{cWl} \\
3 \mathrm{c} \\
100\end{array}$ & $\begin{array}{l}7,652.49 \\
7,640.93 \\
7,620.25 \\
7,618.32 \\
7,611.90\end{array}$ & $\begin{array}{l}13,064.05 \\
13,083.82 \\
13,119.32 \\
13,122.65 \\
13,133.71\end{array}$ & $\begin{array}{r}10-21 \\
10-22 \\
17.1-25\end{array}$ & $\begin{array}{c}2 \\
3 \mathrm{c} \\
2 \mathrm{c} \\
15 \\
3\end{array}$ & $\begin{array}{l}7,431.93 \\
7,416.44 \\
7,413.41 \\
7,409.47 \\
7,404.27\end{array}$ & $\begin{array}{l}13,451.76 \\
13,479.85 \\
13,485.37 \\
13,492.53 \\
13,502.01\end{array}$ & $19-34$ \\
\hline $\begin{array}{l}2 \\
2 \mathrm{c} \\
6 \mathrm{cWW}] \\
200 \mathrm{cw} \\
2 \mathrm{c} ?\end{array}$ & $\begin{array}{l}7,596.30 \\
7,587.66 \\
7,583.26 \\
7,578.72 \\
7,573.47\end{array}$ & $\begin{array}{l}13,160.69 \\
13,175.67 \\
13,183.32 \\
13,191.21 \\
13,200.36\end{array}$ & $101-23$ & $\begin{array}{l}2 \mathrm{c} \\
4 \mathrm{cWv} \\
15 \\
1 \\
2\end{array}$ & $\begin{array}{l}7,396.04 \\
7,390.71 \\
7,386.35 \\
7,384.07 \\
7,382.68\end{array}$ & $\begin{array}{l}13,517.03 \\
13,526.78 \\
13,534.76 \\
13,538.94 \\
13,541.49\end{array}$ & 20. $2-51$ \\
\hline $\begin{array}{l}1 \mathrm{p} ? \\
6 \\
30 \\
5 \mathrm{cWl} \\
1 \mathrm{p} ?\end{array}$ & $\begin{array}{l}7,569.66 \\
7,567.82 \\
7,548.71 \\
7,545.37 \\
7,543.22\end{array}$ & $\begin{array}{l}13,207.00 \\
13,210.21 \\
13,243.65 \\
13,249.52 \\
13,253.29\end{array}$ & & $\begin{array}{l}2 \mathrm{c} \\
2 \\
30 \mathrm{cWl} \\
1 \\
5\end{array}$ & $\begin{array}{r}7,362.12 \\
7,360.95 \\
7,352.03 \\
7,346.88 \\
7,329.37\end{array}$ & $\begin{array}{l}13,579.31 \\
13,581.47 \\
13,597.95 \\
13,607.48\end{array}$ & \\
\hline $\begin{array}{l}1 \mathrm{p} ? \\
2 \\
3\end{array}$ & $\begin{array}{l}7,543.22 \\
7,539.73 \\
7,536.42 \\
7,526.50\end{array}$ & $\begin{array}{l}13,253.29 \\
13,259.43 \\
13,265.25 \\
13,282.73\end{array}$ & & $\begin{array}{l}5 \\
6 \\
2 \\
4 \mathrm{CW}\end{array}$ & $\begin{array}{l}7,329.37 \\
7,324.20 \\
7,318.02 \\
7,316.1\end{array}$ & $\begin{array}{l}13,639.98 \\
13,649.61 \\
13,661.14 \\
13,664.7\end{array}$ & \\
\hline $20 \mathrm{cW}$ & $\begin{array}{l}7,526.50 \\
7,524.48 \\
7,494.04\end{array}$ & $\begin{array}{l}13,282.73 \\
13,286.30 \\
13,340.27\end{array}$ & & $\begin{array}{c}4 \mathrm{c} \\
250 \mathrm{cWl} \text { ? } \\
100 \mathrm{cWl} \\
3\end{array}$ & $\begin{array}{l}7,307.55 \\
7,292.67 \\
7,273.84 \\
7,265.45\end{array}$ & $\begin{array}{l}13,680.72 \\
13,708.63 \\
13,744.12 \\
13,759.99\end{array}$ & $\begin{array}{r}20.3-57 \\
22-67 \\
21-67\end{array}$ \\
\hline $\begin{array}{l}2 \\
5 \mathrm{cw} \\
1 \mathrm{p} ? \\
6 \\
4\end{array}$ & $\begin{array}{l}7,489.13 \\
7,488.12 \\
7,482.62 \\
7,481.15 \\
7,478.63\end{array}$ & $\begin{array}{l}13,349.02 \\
13,350.82 \\
13,360.63 \\
13,363.25 \\
13,367.76\end{array}$ & . & $\begin{array}{c}4 \\
200 \mathrm{cWl} \\
3 \\
20\end{array}$ & $\begin{array}{l}7,263.87 \\
7,246.67 \\
7,237.28 \\
7,228.03\end{array}$ & $\begin{array}{l}13,762.98 \\
13,795.64 \\
13,813.54 \\
13,831.22\end{array}$ & $\begin{array}{l}9-21 \\
9-22\end{array}$ \\
\hline
\end{tabular}

Washington, April 5, 1933. 